\title{
A Rare Finding of Schwannoma of the Vidian Canal: A Case Report
}

\author{
Alisa Yamasaki ${ }^{1,2}$ Ahmad R. Sedaghat ${ }^{1,2}$ Giant C. Lin ${ }^{1,2}$ William T. Curry ${ }^{3}$ Helen A. Shih ${ }^{4}$ \\ Stacey T. Gray ${ }^{1,2}$
}

${ }^{1}$ Department of Otolaryngology-Head and Neck Surgery, Massachusetts Eye and Ear Infirmary, Boston, Massachusetts, United States

2 Department of Otology and Laryngology, Harvard Medical School, Boston, Massachusetts, United States

${ }^{3}$ Department of Neurosurgery, Massachusetts General Hospital, Harvard Medical School, Boston, Massachusetts, United States

${ }^{4}$ Department of Radiation Oncology, Massachusetts General Hospital, Harvard Medical School, Boston, Massachusetts, United States

\author{
Address for correspondence Stacey T. Gray, MD, Department of \\ Otolaryngology, Massachusetts Eye and Ear Infirmary, \\ 243 Charles St, Boston, MA 02114, United States \\ (e-mail: stacey_gray@meei.harvard.edu).
}

J Neurol Surg Rep 2015;76:e48-e51.

\begin{abstract}
Keywords

- vidian canal

- schwannoma

- fractionated radiation therapy

Background Schwannomas of the vidian canal are an extremely rare type of intracranial tumor that can have variable clinical presentations including headache, facial pain, facial muscle paralysis, decreased lacrimation, or nasal dryness. We present an atypical case of an incidentally identified asymptomatic vidian canal schwannoma.

Case Description A 49-year-old woman with a history of multiple sclerosis presented for routine surveillance magnetic resonance imaging that detected an ovoid mass originating in the vidian canal. Given the unusual location of the lesion, an endoscopic endonasal biopsy was performed and confirmed the diagnosis of a vidian canal schwannoma, for which the patient chose to receive fractionated radiation therapy. Conclusion When a vidian canal tumor is identified, endoscopic endonasal biopsy can be used to confirm the diagnosis before pursuing either surgical or radiotherapy treatment. In particular, fractionated radiation therapy offers a radiobiologically safe means of delivering radiation when there is concern for late radiation-related side effects following treatment completion.
\end{abstract}

\section{Background}

Schwannomas are rare tumors of the nerve sheath that arise from the primary glial cells of the peripheral nervous system. As such, schwannomas may arise from any myelinated peripheral nerve. They are generally well-circumscribed lesions that rarely undergo malignant degeneration. Although benign and slow growing, schwannomas can cause significant morbidity due to direct involvement of nerves or by local mass effect that can pose particular challenges for treatment.

Approximately 25 to $45 \%$ of all schwannomas are found in the head and neck, where they can originate from virtually any cranial nerve. ${ }^{1}$ The most common schwannoma is the vestibular schwannoma that arises from the vestibular branch of the eighth cranial nerve and accounts for $51 \%$ of all nerve sheath tumors found anywhere in the body. ${ }^{2}$ This is followed by schwannomas of the trigeminal nerve that comprise up to $8 \%$ of all schwannomas. ${ }^{3}$ By contrast, schwannomas in the nasal cavity or paranasal sinusesand vidian canal schwannomas in particular-are much rarer. The vidian canal, also referred to as the pterygoid canal, extends from the pterygopalatine fossa to the foramen lacerum in the floor of the sphenoid sinus ${ }^{4}$ and contains the vidian artery as well as the vidian nerve. The vidian received

August 1, 2014

accepted

October 1, 2014

published online

February 5, 2015
DOI http://dx.doi.org/

10.1055/s-0034-1544112.

ISSN 2193-6366. (c) 2015 Georg Thieme Verlag KG

Stuttgart · New York
License terms

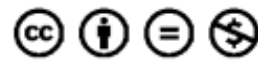



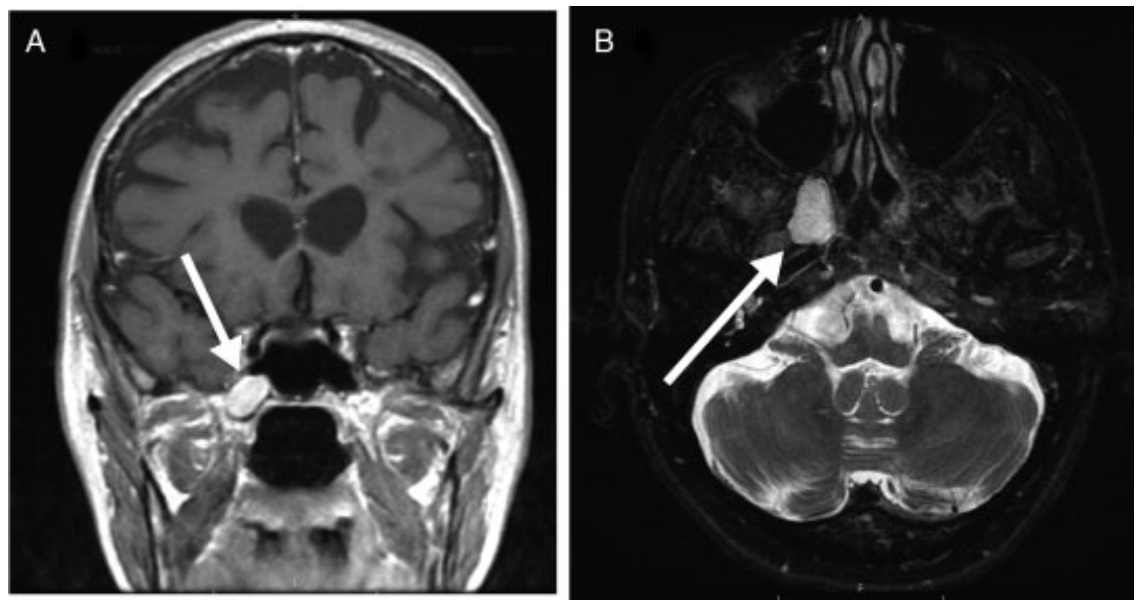

Fig. 1 An ovoid $14 \times 20 \times 13$-mm mass (white arrows) centered in an enlarged right vidian canal and extending along the inferior margin of the right cavernous sinus. The mass has (A) bright T1 signal post contrast, coronal view, and (B) bright T2 signal with fat saturation. There was no evidence of diffusion restriction (not shown).

nerve arises from the joining of the greater superficial petrosal nerve and the deep petrosal nerve. The greater superficial petrosal nerve consists of preganglionic parasympathetic fibers from the geniculate ganglion of the facial nerve. The deep petrosal nerve consists of postganglionic sympathetic fibers from the carotid sympathetic plexus. ${ }^{5-8}$ The parasympathetic fibers of the vidian nerve synapse in the pterygopalatine ganglion, whereas the sympathetic fibers innervate the lacrimal gland and secretory pathways of the nasopalatine glands.,

Schwannomas of the vidian canal can present with a variety of clinical symptoms. Damage to the vidian nerve itself can result in decreased or absent lacrimation, corneal desiccation as well as dryness of the nasal mucosa, and cluster headaches. ${ }^{5}$ Additionally, vidian schwannomas have been reported to compress the adjacent internal carotid artery or bulge through the lateral wall of the sphenoid sinus to expand into the cavernous sinus. 6 ,

To date, only 4 cases of vidian nerve schwannomas and 11 cases of histologically unconfirmed peri-vidian tumors have been reported in the literature. ${ }^{10-15}$ Here we present a case of biopsy-proven schwannoma of the vidian canal. We discuss the patient's initial presentation, imaging characteristics, histologic findings, and treatment for this unusual tumor and review the other reported cases of vidian canal schwannomas in the literature.

\section{Case History}

The patient is a 49-year-old woman with a 24-year history of multiple sclerosis (MS) who presented for follow-up evaluation after an acute flare of MS. In the past, her MS flares had been relatively infrequent, with motor and/or peripheral sensory deficits that were well controlled with short-term courses of steroids. During her most recent flare, she experienced numbness of her left head, arm, and leg, with full resolution of symptoms after steroid treatment. Her past medical history was otherwise unremarkable.
A routine surveillance brain magnetic resonance imaging (MRI) was performed to evaluate for MS progression. MRI showed minimal progression of disease compared with imaging from 8 years prior, with extensive T2, fluid-attenuated inversion recovery, and proton-density prolonged signal in the periventricular, pericallosal, and subcortical white matter consistent with MS. Incidentally, the MRI also revealed a $19 \times 13 \times 10 \mathrm{~mm}$ ovoid mass along the inferior margin of the right cavernous sinus that was not present on previous MRIs. The lesion was well circumscribed and homogeneously enhancing, with $\mathrm{T} 1$ hypointense and $\mathrm{T} 2$ hyperintense signal. A repeat dedicated MRI was performed that demonstrated the lesion originated within an expanded vidian canal ( $\mathbf{- F i g . 1}$ ). A sinus computed tomography scan also showed bony erosion of an expanded vidian canal (-Fig. 2). From a symptom standpoint, the patient noted some mild nasal congestion in the mornings but was otherwise asymptomatic without any facial pain or paresthesias, abnormal lacrimation, or dry eye symptoms. Physical examination was similarly unremarkable with a normal nasal endoscopy and no focal neurologic deficits. An endoscopic

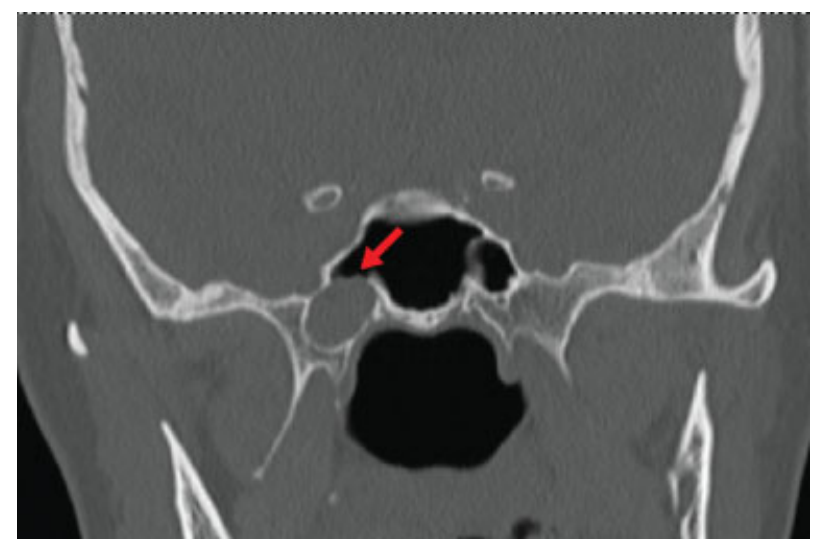

Fig. 2 Preoperative sinus computed tomography scan without contrast demonstrating bony erosion of the right vidian canal (red arrow). 
endonasal biopsy was performed with image guidance. The sphenoid ostium was identified medial to the superior turbinate in a transethmoidal fashion. The ostium was enlarged laterally and inferiorly, and a wide sphenoidotomy was created. The mass was easily identified within a noticeably expanded vidian canal along the lateral floor of the sphenoid sinus (-Fig. 3). The canal was opened along the floor of the sphenoid sinus to uncover the mass and a biopsy was obtained. The final histopathologic assessment confirmed a diagnosis of schwannoma. The patient's recovery was unremarkable, and she had no problems with dry eye or nasal irritation postoperatively. Two weeks after surgery, her endoscopic examination showed a well-healed sinus cavity.

Options of endoscopic surgical resection, single-fraction stereotactic radiosurgery (SRS), and conventional fractionated radiotherapy using stereotactic radiotherapy (SRT) were reviewed with the patient, and she decided to pursue treatment with radiation. Given the benign histology, a background of MS that could confer additional sensitivity to radiation, and the patient's young age, the patient decided to pursue SRT to minimize late normal tissue injury risks. SRT was delivered at $1.8 \mathrm{~Gy}$ per fraction to $50.4 \mathrm{~Gy}$. Treatment was well tolerated; the most notable symptom was mild fatigue.

\section{Discussion}

Schwannomas of the vidian canal are an extremely rare type of intracranial tumor with variable presentation. Symptoms of vidian schwannomas can result from compression of surrounding structures or extension into surrounding areas that can cause headaches, facial pain and paresthesias, and facial muscle paralysis. Dysfunction of the vidian nerve itself may cause nasal dryness, decreased lacrimation, and corneal desiccation. Here we present an unusual case of a vidian nerve schwannoma incidentally identified in a patient who was asymptomatic from the lesion.

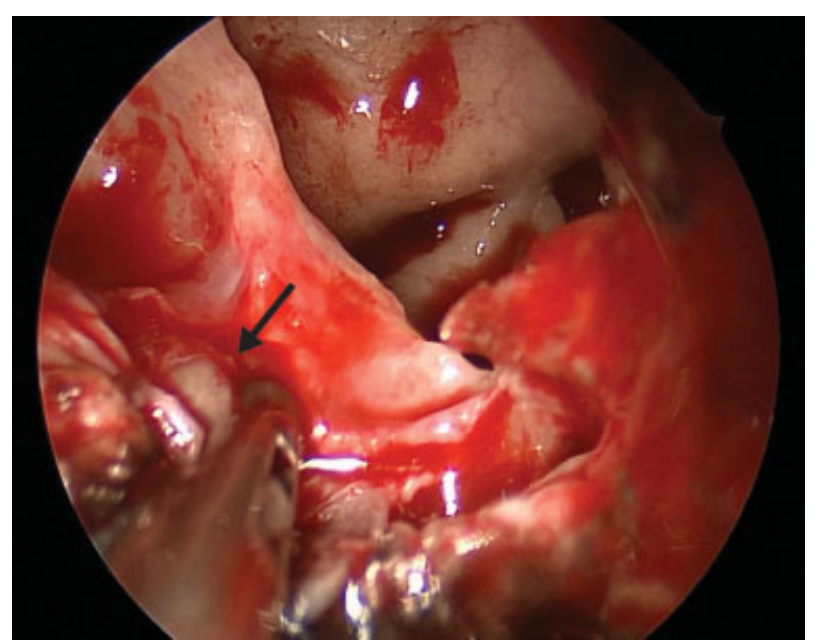

Fig. 3 Intraoperative endoscopic views of biopsy using transsphenoidal approach. The mass (black arrow) is visible protruding from the right vidian canal, located at the floor of the sphenoid sinus.
To date, four documented cases of biopsy-proven vidian nerve schwannomas have appeared in the literature. Reported cases include both male and female patients, ranging in age from 13 to 78 years, with both unilateral and bilateral lesions described. ${ }^{10-15}$ Presenting symptoms included headache and facial palsy, oculomotor palsy and cerebrospinal fluid leak, unilateral hearing loss secondary to obstructive serous otitis media, and facial paresthesias. Complete surgical resection was pursued for all four cases through both open and endonasal approaches, although one patient with bilateral vidian nerve schwannomas had only partial resection of one lesion due to difficulties with surgical access. ${ }^{13}$ All patients had significantly improved or stable symptoms, and the two patients with the most severe symptoms-one with oculomotor palsy and ptosis and the other with headache and facial nerve palsy-demonstrated the most dramatic postoperative improvement with near to complete resolution of symptoms. ${ }^{10,13}$

Consistent with these cases, the traditional and curative management of peripheral nerve tumors is surgical; recent advancements in transnasal endoscopic skull base approaches frequently enable complete resection. Radiation therapy by either SRS or multiple fraction radiotherapy is also an alternative option for treatment. Conservative management through observation with serial imaging is also reasonable when surgical resection is either not feasible or involves a disproportionate risk of postsurgical morbidity and/or mortality. The choice of treatment modality depends on patient choice, functional status, presenting symptoms, tumor location, tumor volume, and-in rare instances-malignant potential. The ultimate goal of both surgery and radiotherapy is to maximize tumor control while also optimizing preservation of surrounding structures.

In this case, the patient decided to pursue treatment with radiation therapy. Conservative management was not considered, given the significant increase in tumor volume during the eight-year interval of MRI surveillance and the presence of bone erosion. Given the rare presentation of a mass located in the vidian canal, the decision was made to confirm the diagnosis with a biopsy prior to treatment. After histologic confirmation, the schwannoma was deemed favorable to be controlled by radiation. The choice between single-fraction SRS or multiple-fraction radiation was made based on size and proximity of the tumor to potential radiation-sensitive structures. Minimizing the risk of adverse effects from radiation was the determinant for pursuing fractionated radiation.

In recent years, radiation therapy has become a common alternative to surgical resection of intracranial schwannomas. ${ }^{16-21}$ Options include either single-fraction SRS or conventional daily low-dose fractionation over approximately five weeks. SRS is a well-tolerated procedure, typically completed during a single session of irradiation and far more convenient for the patient. SRS has become a widely accepted treatment option for vestibular schwannomas and has a high success rate of durable tumor control with often partial reduction in tumor volume, with progression-free survival of $93 \%$ at 5 years and $92 \%$ at 10 years. ${ }^{22}$ Fractionated radiation has similar results and is chosen over SRS when there is a concern that the size of target 
exceeds safe tolerance to SRS, when there are critical radiationsensitive structures in adjacent areas that may not be able to tolerate the single high-dose collateral exposure, or when there are underlying concerns of the patient to suggest a higher risk of an adverse event. Compared with surgery, radiation therapy has been reported in several studies to result in decreased mortality and fewer treatment-related complications for vestibular schwannoma including hearing loss and facial weakness. ${ }^{22-25}$ Recent advances in imaging and radiation delivery offer more conformal treatment options such as SRT-which combines both the superior accuracy of target localization of a stereotactic system and reduction in the size of the radiation treatment fields-and fractionated treatment, a radiobiologically safer means of delivering radiation when there are concerns for late effects of radiation decades after treatment completion.

\section{Conclusion}

Schwannomas of the vidian canal are extremely rare tumors and may present with symptoms of headaches, facial pain, facial muscle paralysis, decreased lacrimation, and nasal dryness or as an incidental finding. When a vidian canal tumor is identified, endoscopic biopsy can be used to confirm a histopathologically benign diagnosis before considering treatment options. Treatment options include surgical resection and radiotherapy.

\section{References}

1 Malone JP, Lee W-J, Levin RJ. Clinical characteristics and treatment outcome for nonvestibular schwannomas of the head and neck. Am J Otolaryngol 2005;26(2):108-112

2 Propp JM, McCarthy BJ, Davis FG, Preston-Martin S. Descriptive epidemiology of vestibular schwannomas. Neuro Oncol 2006; 8(1):1-11

3 Samii M, Migliori MM, Tatagiba M, Babu R. Surgical treatment of trigeminal schwannomas. J Neurosurg 1995;82(5):711-718

4 Jacopo G, Micaela I, Italo C, Luigi C, Larocca LM, Gaetano P. Atypical sinonasal Schwannomas: a difficult diagnostic challenge. Auris Nasus Larynx 2009;36(4):482-486

5 Tubbs RS, Salter EG. Vidius Vidius (Guido Guidi): 1509-1569. Neurosurgery 2006;59(1):201-203; discussion 201-203

6 Osawa S, Rhoton AL Jr, Seker A, Shimizu S, Fujii K, Kassam AB. Microsurgical and endoscopic anatomy of the vidian canal. Neurosurgery 2009;64(5, Suppl 2):385-411, discussion 411-412

7 Konno A. Historical, pathophysiological, and therapeutic aspects of vidian neurectomy. Curr Allergy Asthma Rep 2010;10(2): 105-112

8 Waxman SG. The Autonomic Nervous System. In: Waxman SG, ed. Clinical Neuroanatomy. 27th ed. New York, NY: McGraw-Hill; 2013:241-255
9 Unal B, Bademci G, Bilgili YK, Batay F, Avci E. Risky anatomic variations of sphenoid sinus for surgery. Surg Radiol Anat 2006; 28(2):195-201

10 Wu SW, Chen WL, Chen WL, Chen MK. Transnasal endoscopic resection of vidian nerve schwannoma accompanied by sphenoid mucopyocele and oculomotor palsy: a case report. B-ENT 2012; 8(3):207-211

11 Hackman T, Rickert CG, Getz AE, Uppaluri R. Endoscopic surgical management of vidian nerve schwannoma. Laryngoscope 2011; 121(2):241-244

12 Honda K, Asato R, Tanaka S, Endo T, Nishimura K, Ito J. Vidian nerve schwannoma with middle cranial fossa extension resected via a maxillary swing approach. Head Neck 2008; 30(10):1389-1393

13 Cheong JH, Kim JM, Bak KH, Kim CH, Oh YH, Park DW. Bilateral vidian nerve schwannomas associated with facial palsy. Case report and review of the literature. J Neurosurg 2006;104(5): 835-839

14 Blandino A, Gaeta M, Minutoli F, Pandolfo I. CT and MR findings in neoplastic perineural spread along the vidian nerve. Eur Radiol 2000;10(3):521-526

15 Teresi LM, Lufkin RB, Viñuela F, et al. MR imaging of the nasopharynx and floor of the middle cranial fossa. Part II. Malignant tumors. Radiology 1987;164(3):817-821

16 Hasegawa T. Stereotactic radiosurgery for nonvestibular schwannomas. Neurosurg Clin N Am 2013;24(4):531-542

17 Konovalov AN, Spallone A, Mukhamedjanov DJ, Tcherekajev VA, Makhmudov UB. Trigeminal neurinomas. A series of 111 surgical cases from a single institution. Acta Neurochir (Wien) 1996; 138(9):1027-1035

18 McCormick PC, Bello JA, Post KD. Trigeminal schwannoma. Surgical series of 14 cases with review of the literature. J Neurosurg 1988;69(6):850-860

19 Mulder JJ, Kaanders JH, van Overbeeke JJ, Cremers CW. Radiation therapy for vestibular schwannomas. Curr Opin Otolaryngol Head Neck Surg 2012;20(5):367-371

20 Arthurs BJ, Fairbanks RK, Demakas JJ, et al. A review of treatment modalities for vestibular schwannoma. Neurosurg Rev 2011; 34(3):265-277; discussion 277-279

21 Murphy ES, Suh JH. Radiotherapy for vestibular schwannomas: a critical review. Int J Radiat Oncol Biol Phys 2011;79(4):985-997

22 Hasegawa T, Kida Y, Kato T, Iizuka H, Kuramitsu S, Yamamoto T. Long-term safety and efficacy of stereotactic radiosurgery for vestibular schwannomas: evaluation of 440 patients more than 10 years after treatment with Gamma Knife surgery. J Neurosurg 2013;118(3):557-565

23 McClelland S III, Guo H, Okuyemi KS. Morbidity and mortality following acoustic neuroma excision in the United States: analysis of racial disparities during a decade in the radiosurgery era. Neuro Oncol 2011;13(11):1252-1259

24 Pollock BE, Driscoll CL, Foote RL, et al. Patient outcomes after vestibular schwannoma management: a prospective comparison of microsurgical resection and stereotactic radiosurgery. Neurosurgery 2006;59(1):77-85; discussion 77-85

25 Kondziolka D, Lunsford LD, McLaughlin MR, Flickinger JC. Longterm outcomes after radiosurgery for acoustic neuromas. N Engl J Med 1998;339(20):1426-1433 